\title{
Integrating multiple sources of biodiversity information greatly expands the range of a rare species of Hymenoptera (Vanhorniidae)
}

\author{
Joshua Hogan ${ }^{\ddagger}$, Amber I.H. Bass ${ }^{\ddagger}$, Y. Miles Zhang ${ }^{\S}$, Barbara J Sharanowski ${ }^{\ddagger}$ \\ ‡ University of Central Florida, Orlando, United States of America \\ $\S$ University of Florida, Gainesville, United States of America
}

Corresponding author: Barbara J Sharanowski (barb.sharanowski@ucf.edu)

Academic editor: Yasen Mutafchiev

Received: 21 Jun 2019 | Accepted: 12 Jul 2019 | Published: 16 Jul 2019

Citation: Hogan J, Bass Al.H, Zhang YM, Sharanowski BJ (2019) Integrating multiple sources of biodiversity information greatly expands the range of a rare species of Hymenoptera (Vanhorniidae). Biodiversity Data Journal 7: e37569. https://doi.org/10.3897/BDJ.7.e37569

\begin{abstract}
Background

Vanhornia eucnemidarum Crawford is the only species of Vanhorniidae that occurs in North America. This species is rarely collected and thus the distribution is not well documented. Intending to uncover a more accurate range of this species, we assembled collection records from museums, personal collections and citizen science projects. Many of these records were non-digitised and had to be personally requested.
\end{abstract}

\section{New information}

Here we expand the known distribution of $V$. eucnemidarum to include nine new provinces and states: Manitoba, Connecticut, Oregon, Mississippi, Missouri, New Hampshire, New Jersey, Texas and Wisconsin. Although Quebec has been listed as a previous locality, the recorded province was mislabelled, so Quebec is now also officially a provincial record. 


\section{Keywords}

Proctotrupoidea, parasitoid, Eucnemidae, Isorhipis, citizen science, museum science, databasing, distribution, Vanhornia eucnemidarum, digitisation

\section{Introduction}

In spite of the fact that many species on our planet remain undescribed (Scheffers et al. 2012), our in-depth knowledge of described species is often lacking in basic biology, ecology or geographic range information. With increasing efforts in the digitisation of georeferenced specimens, we expand our understanding of both described species and of overall global biodiversity (Monfils et al. 2017). There is often a wealth of information stored within smaller non-digitised collections and citizen science platforms (Ridenbaugh et al. 2018, Silvertown 2009, Skvarla et al. 2015). When collated, these sources can greatly increase knowledge on species, particularly with respect to geographic range. This highlights the importance of museums and specimen digitisation (Mehrhoff 1997), as well as the valuable part that the general public can play in expanding our knowledge of species (Ellwood et al. 2015), whether previously described or not.

Vanhorniidae (Hymenoptera: Proctotrupoidea, Fig. 1A) are uncommonly collected parasitoids of false click beetle larvae (Coleoptera: Eucnemidae). They can be distinguished from other proctotrupoids using a combination of characters: exodont mandibles (Fig. 1D), low attachment of the antennae (Fig. 1C), deeply pitted mesosoma (Fig. 1B) and long exserted ovipositor, projecting anteriorly from the base and housed in a ventral groove (Champlain 1922, Crawford 1909, Deyrup 1985, Haines 2008, Johnson 2017, Keim 2017, Kleiner et al. 2019, Townes and Townes 1981). Vanhorniidae contains a single genus Vanhornia Crawford and includes three described species, V. eucnemidarum Crawford, 1909, V. quizhouensis (He \& Chu, 1990) and V. leileri Hedquist, 1976 (Crawford 1909, He and Chu 1990, Hedqvist 1976, Kozlov 1998). Vanhornia eucnemidarum is the only North American species, with a known range including the states of Florida, Georgia, Kansas, Kentucky, Maine, Maryland, Michigan, New York, North Carolina, Ohio, South Carolina, Tennessee, Virginia and West Virginia and the Canadian provinces of Ontario and Quebec (Champlain 1922, Crawford 1909, Deyrup 1985, Haines 2008, Johnson 2017 , Keim 2017, Kleiner et al. 2019, Townes and Townes 1981). Along with North American records of $V$. eucnemidarum, this species has also been recorded in South Korea (Choi and Lee 2012).

Little is known about the host use and host breadth of this species. The type specimen of $V$. eucnemidarum was collected from the larval gallery of an unknown species of false click beetle (Crawford 1909). It has since been reared from the larval/pupal galleries of Isorhipis ruficornis (Say, 1823) found in dead maple (Brues 1927, Champlain 1922), with one record specifically from the sugar maple (Deyrup 1985). Vanhornia eucnemidarum may also be associated with beech (Smith 1995), another known host plant of I. ruficornis (Brues 1927). 


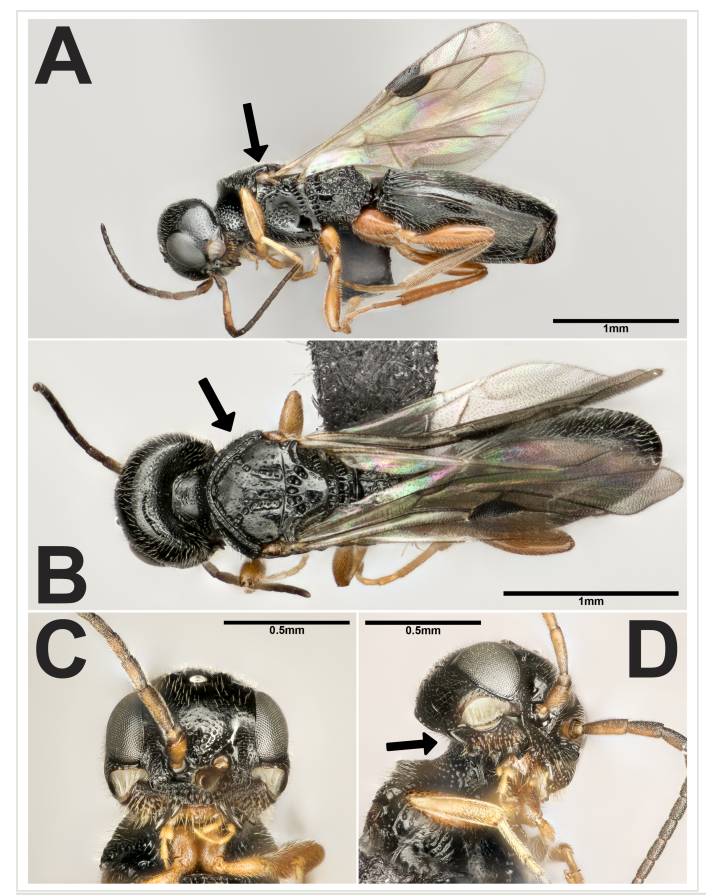

Figure 1. doi

Vanhornia eucnemidarum female collected from Howden, Manitoba, Canada. A. Lateral habitus, showing brown tegula at base of wing; B. Dorsal habitus, showing deeply pitted mesosoma; C. Head anterior view; D. Head, antero-ventral view to show exodont mandibles.

Within Canada, V. eucnemidarum was previously recorded from Ontario and Quebec, however Townes and Townes (1981) mislabelled the Quebec specimen, which was actually found in Ontario. We collected two $V$. eucnemidarum specimens from a malaise trap in Manitoba, representing a new provincial record for Canada. This subsequently led to a search of museum records, citizen science websites and communications with professional and amateur entomologists for any unreported collections. The objective of this paper is to report all new state and provincial records of $V$. eucnemidarum to better understand its North American distribution.

\section{Materials and methods}

Two specimens of $V$. eucnemidarum were collected by malaise trap in Howden, Manitoba (49.734996, -97.129860) between the dates of 7-14 August 2015 in a stand of trees that included maple. Additional collection records were gathered from personal communications with museums (Table 1) and collectors, online databases and the online citizen science community BugGuide (Haines 2008, Johnson 2017, Keim 2017). The majority of personal communications were facilitated through the Entomological Collections Network listserv 
(ECN-L). Accessed databases include iDigBio (Integrated Digitized Biocollections), DiscoverLife, SCAN (Symbiota Collections of Arthropods Network), BISON (Biodiversity Information Serving Our Nation) and GBIF (Global Biodiversity Information Facility).

Table 1.

Listed are all collections referenced in this manuscript and supplemental along with their associated acronyms

\begin{tabular}{|c|c|}
\hline Acronym & Name of Collection \\
\hline AMNH & American Museum of Natural History \\
\hline CAS & California Academy of Sciences \\
\hline $\mathrm{CNCl}$ & Canadian National Collection of Insects \\
\hline DEBU & University of Guelph \\
\hline LEMQ & Lyman Entomological Museum \\
\hline FSCA & Florida State Collection of Arthropods \\
\hline MCZC & Museum of Comparative Zoology \\
\hline MEM & Mississippi State University \\
\hline MSUC & Michigan State University \\
\hline NCSU & North Carolina State University \\
\hline PMAE & Royal Alberta Museum \\
\hline PMNH & Peabody Museum of Natural History \\
\hline INHS & Illinois Natural History Survey \\
\hline OSUC & C.A. Triplehorn Insect Collection \\
\hline QMOR & Collection Entomologique Ouellet-Robert \\
\hline RMNH & Naturalis Biodiversity Centre \\
\hline ROME & Royal Ontario Museum \\
\hline SEMC & Snow Entomological Museum \\
\hline TAMU & Texas A \& M University \\
\hline UCFC & University of Central Florida \\
\hline UMMZ & University of Michigan \\
\hline UMSP & University of Minnesota \\
\hline UNHC & University of New Hampshire \\
\hline WIRC & University of Wisconsin \\
\hline WRME & Wallis Roughley Museum of Entomology \\
\hline
\end{tabular}


All locality records have been entered in Darwin Core archive format (Suppl. material 1). Any locality records that lacked geographic coordinates were input into Google Maps, generating a close approximation of the collection site (these coordinates were used for mapping, but not archived). Collection events were mapped using Simplemappr (Shorthouse 2010) with new state and provincial records indicated (Fig. 2).

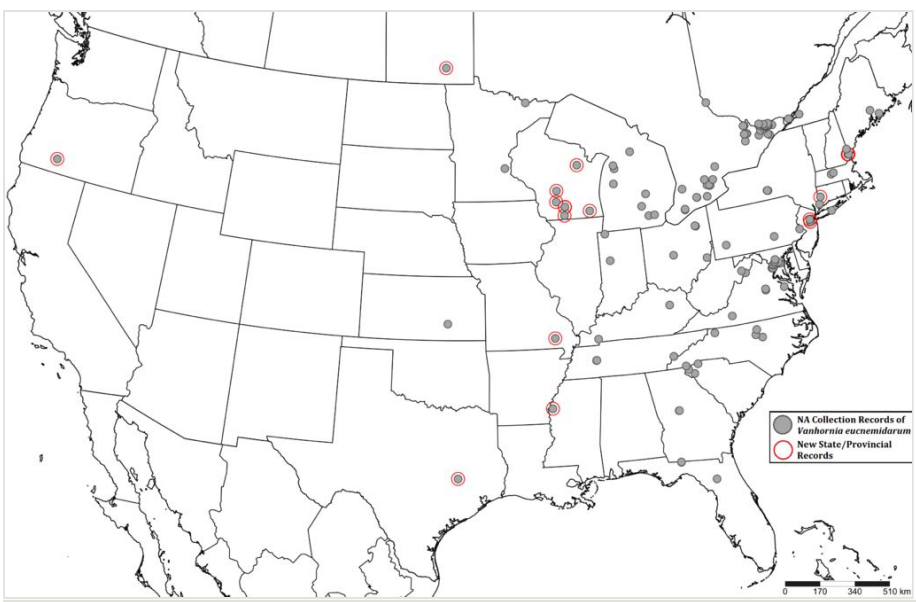

Figure 2. doi

Range map of Vanhornia eucnemidarum in North America. Grey circles represent all North American collection records gathered during the study. Those records surrounded by a red circle represent new state/provincial records.

All photos in this study were taken using a Canon 7D Mark II with either a Canon MP-E 65 mm F/2.8 Macro photo lens or a Mitutoyo M Plan Apo 10x objective mounted on to the Canon EF Telephoto 70-200 mm zoom lens. Multiple images were taken across numerous focal planes and combined using Zerene Stacker 1.04. Images were edited using Adobe Photoshop CC and plates were prepared using Adobe Illustrator CC.

\section{Taxon treatment}

\section{Vanhornia eucnemidarum Crawford 1909}

- Catalogue of Life http://www.catalogueoflife.org/col/details/species/ id/84c6010b1c8cef32c9a7ee8e730446b8

\section{Materials}

a. ScientificName: Vanhornia eucnemidarum Crawford 1909; kingdom: Animalia; phylum: Arthropoda; class: Insecta; order: Hymenoptera; family: Vanhorniidae; taxonRank: species; genus: Vanhornia; scientificNameAuthorship: Crawford 1909; continent: North America; country: Canada; countryCode: CA; stateProvince: Manitoba; verbatimLocality: Howden; geodeticDatum: WGS84; samplingProtocol: Malaise Trap; eventDate: 2015-07-14; year: 2015; month: 07; day: 14; sex: female; lifeStage: adult; 
catalogNumber: JBWM0378038; recordedBy: Amber Bass; institutionCode: WRME;

basisOfRecord: PreservedSpecimen; occurrenceID: UCFC:Veuc:00000265

b. $\quad$ scientificName: Vanhornia eucnemidarum Crawford 1909; kingdom: Animalia; phylum: Arthropoda; class: Insecta; order: Hymenoptera; family: Vanhorniidae; taxonRank: species; genus: Vanhornia; scientificNameAuthorship: Crawford 1909; continent: North America; country: Canada; countryCode: CA; stateProvince: Manitoba; verbatimLocality: Howden; geodeticDatum: WGS84; samplingProtocol: Malaise Trap; eventDate: 2015-07-14; year: 2015; month: 07; day: 14; sex: female; lifeStage: adult; catalogNumber: UCFC0528248; recordedBy: Amber Bass; institutionCode: UCFC; basisOfRecord: PreservedSpecimen; occurrenceID: UCFC:Veuc:00000196

\section{Diagnosis}

Vanhornia eucnemidarum can be distinguished from other species of Vanhornia by the following combination of characters: Antennal sockets inserted immediately above dorsal margin of clypeus; tegulae black to dark brown but never yellow; and rugulose metasomal striations restricted to the basal third.

\section{Discussion}

\section{New records and possible host associations}

Our search discovered 278 specimen records and three BugGuide photos. These data represent new records for $V$. eucnemidarum in the states of Connecticut, Mississippi, Missouri, New Hampshire, New Jersey, Oregon, Texas and Wisconsin in USA and the Canadian province of Manitoba (Fig. 2). Additionally, we present high-quality montaged photos of $V$. eucnemidarum (Fig. 1), illustrating important distinguishing features, unique to this species such as the exodont mandibles (Fig. 1D). The new records reported in this study expand the range of $V$. eucnemidarum north to Manitoba, west to Oregon and south to Texas. This vast increase in the known range of $V$. eucnemidarum may be due to rarity of collection (Deyrup 1985), lack of study or lack of recognition by non-specialists.

Though the new records of $V$. eucnemidarum, presented here, do not include host associations, they do allow for some speculation regarding the only known host, $I$. ruficornis. Several records of $V$. eucnemidarum were found to be in a state or province in which the known host $I$. ruficornis has not yet been recorded (Otto and Karns 2017). These include the states of Florida, Kentucky, Maine, Mississippi, Oregon, South Carolina, Tennessee and West Virginia, along with the Canadian province of Manitoba. This suggests that one of two things may be true: I. ruficornis may be present in these areas but has not yet been collected or $V$. eucnemidarum may have a wider host breadth than previously thought. If the latter is true, we suspect the host range may include other Isorhipis species.

The only known host plant associations of $V$. eucnemidarum are with maple and beech (Brues 1927, Champlain 1922, Smith 1995). Isorhipis ruficornis is a generalist on dead and rotting wood and, in addition to maple and beech, this species has been known to 
associate with eastern hemlock (Buck et al. 2005) and elm (Hoffmann 1942). Targeted sampling and rearing of $V$. eucnemidarum across a range of possible hosts will provide more information about the host breadth of $V$. eucnemidarum and may help predict its full range and possible biological control potential.

\section{Importance of museum collections and citizen science in biodiversity studies}

Biodiversity studies, such as this one, assist in building more complete ranges for species, which are vital for ecological, evolutionary and applied biological research. Researchers often have limited access to these data, with an estimated $10 \%$ of specimen data stored in a digital form and even less made available online (Page et al. 2015). Only $22(\sim 15.4 \%)$ of the 143 novel locality records gathered during this study were previously listed in online databases. This is unsurprising as biodiversity collections and their efforts to database and digitise specimen data have historically been undervalued and underfunded, despite research showing funding for such efforts could save the government and taxpayers thousands of dollars in research costs by eliminating redundancy and allowing scientists easy access to the information they require (Lavoie 2013, Monfils et al. 2017, Suarez and Tsutsui 2004, Thiers et al. 2019).

Citizen science records provided another important source of locality data used in this study. Photos, uploaded through citizen science projects and social media websites such as BugGuide, Flickr and iNaturalist, are becoming frequent sources of legitimate taxonomic records (Ridenbaugh et al. 2018, Dickinson et al. 2012, Skvarla et al. 2015, Silvertown 2009, Thiers et al. 2019). This further demonstrates the importance of the unique natural history observations citizens provide researchers.

\section{Conclusions}

This study has updated the distribution records of $V$. eucnemidarum, using a combination of museum, citizen science and digitised records. Given the large increase in range discovered through this study, it is possible that this species is present throughout North America. We hope this paper will aid in recognition of $V$. eucnemidarum by curators and naturalists, which will further our understanding of this enigmatic family of parasitoid wasps.

\section{Acknowledgements}

We thank the following individuals and their respective museums for providing locality information and/or allowing us to examine specimens located in their respective collections: J. Carpenter \& C. Lebeau of AMNH, B. Zuparko of CAS, A. Bennett, M. Locke, \& A. Woodward of $\mathrm{CNCl}$, S. Paiero of DEBU, M. Jackson of LEMQ, E. Talamas of FSCA, R. Hawkins of MCZC, R. Brown of MEM, G. Parsons of MSUC, B. Blinn of NCSU, M. Buck of PMAE, L. Gall of PMNH, C. Dietrich of INHS, N. Johnson of OSUC, E. Normandin of QMOR, F. Bakker of RMNH, A. Guidotti of ROME, J. Thomas of SEMC, J. Oswald, J. 
Woolley, \& K. Wright of TAMU, S. Kelly of UCFC, E. Tucker of UMMZ, R. Holzanthal \& R. Thomson of UMSP, I. Miko of UNHC, C. Brabant of WIRC, J. Gibbs \& L. Irwin of WRME, L. Barringer of the Bureau of Plant Industry, D. Johnson and M. Skvarla for sharing data contained within their respective personal collections, R. Ridenbaugh imaging assistance and D. Dal Pos and E. Talamas for manuscript revisions. Additional thanks go to the reviewers for their thoughtful comments and suggestions.

\section{References}

- $\quad$ Brues C (1927) Observations on wood-boring insects, their parasites and other associated insects. Psyche: A Journal of Entomology 34 (2): 73-90. https://

doi.org/10.1155/1927/78738

- $\quad$ Buck L, Lambdin P, Paulsen D, Grant J, Saxton A (2005) Insect species associated with eastern hemlock in the great smoky mountains national park and environs. Journal of the Tennessee Academy of Science 80 (3-4): 60-69. URL: http://bit.ly/2WTQkNZ

- Champlain AB (1922) Records of hymenopterous parasites in Pennsylvania. Psyche: A Journal of Entomology 29 (3): 95-100. https://doi.org/10.1155/1922/98647

- $\quad$ Choi MB, Lee JW (2012) First record of Vanhorniidae (Hymenoptera: Proctotrupoidea) from Korea. Journal of Asia-Pacific Entomology 15 (1): 59-61. https://doi.org/10.1016/ j.aspen.2011.08.003

- Crawford JC (1909) A new family of parasitic Hymenoptera . Proceedings of the Entomological Society of Washington 11: 63-65.

- $\quad$ Deyrup M (1985) Notes on the Vanhorniidae (Hymenoptera). The Great Lakes Entomologist 18 (2): 65-68. URL: bit.ly/2la8X36

- $\quad$ Dickinson JL, Shirk J, Bonter D, Bonney R, Crain RL, Martin J, Phillips T, Purcell K (2012) The current state of citizen science as a tool for ecological research and public engagement. Frontiers in Ecology and the Environment 10 (6): 291-297. https:// doi.org/10.1890/110236

- $\quad$ Ellwood ER, Dunckel BA, Flemons P, Guralnick R, Nelson G, Newman G, Newman S, Paul D, Riccardi G, Rios N (2015) Accelerating the digitization of biodiversity research specimens through online public participation. BioScience 65 (4): 383-396. https:// doi.org/10.1093/biosci/biv005

- Haines D (2008) Vanhornia eucnemidarum. http://bit.ly/2WOCnvJ. Accessed on: 2018-8-14.

- Hedqvist K (1976) Vanhornia leileri n. sp. from central Sweden (Hymenoptera: Proctotrupidae, Vanhorniinae). Insect Systematics \& Evolution 7 (4): 315-316. https:// doi.org/10.1163/187631276X00540

- $\quad$ He J, Chu J (1990) A new genus and species of Vanhorniidae from China (Hymenoptera: Serphidae). Acta Entomologica Sinica 33: 102-104.

- Hoffmann $\mathrm{CH}$ (1942) Annotated list of elm insects in the United States. 462. United States Department of Agriculture Micellaneous Publication No. 466 URL: http://bit.ly/2XWcFqB

- Johnson R (2017) Vanhornia eucnemidarum. http://bit.ly/31lkxhu. Accessed on: 2018-8-14.

- Keim B (2017) Vanhornia eucnemidarum. http://bit.ly/2KYIPhn. Accessed on: 2018-8-14. 
- $\quad$ Kleiner KN, Hanson P, Pickering J (2019) First record of Vanhorniidae (Hymenoptera: Proctotrupoidae) from Florida. Florida Entomologist 102 (1): 257-258. https:// doi.org/10.1653/024.102.0147

- $\quad$ Kozlov MA (1998) Family Vanhorniidae . A Key to Insects of the Russian Far East 4 (3). [In Russian].

- Lavoie C (2013) Biological collections in an ever changing world: herbaria as tools for biogeographical and environmental studies. Perspectives in Plant Ecology, Evolution and Systematics 15 (1): 68-76. https://doi.org/10.1016/i.ppees.2012.10.002

- $\quad$ Mehrhoff LJ (1997) Museums, research collections, and the biodiversity challenge. Biodiversity II: Understanding and Protecting Our Biological Resources 447-464. https:// doi.org/10.17226/4901

- $\quad$ Monfils AK, Powers KE, Marshall CJ, Martine CT, Smith JF, Prather LA (2017) Natural history collections: teaching about biodiversity across time, space, and digital platforms. Southeastern Naturalist 16 (sp10): 47-58. https://doi.org/10.1656/058.016.0sp1008

- $\quad$ Otto R, Karns K (2017) New state records for Nearctic false click beetles (Coleoptera: Eucnemidae). Insecta Mundi 0582: 1-21. URL: http://bit.ly/2loA3rp

- $\quad$ Page LM, MacFadden BJ, Fortes JA, Soltis PS, Riccardi G (2015) Digitization of biodiversity collections reveals biggest data on biodiversity. BioScience 65 (9): 841-842. https://doi.org/10.1093/biosci/biv104

- $\quad$ Ridenbaugh RD, Barbeau E, Sharanowski BJ (2018) Description of four new species of Eadya (Hymenoptera, Braconidae), parasitoids of the eucalyptus tortoise beetle (Paropsis charybdis) and other eucalyptus defoliating leaf beetles. Journal of Hymenoptera Research 64: 141-175. https://doi.org/10.3897/jhr.64.24282

- $\quad$ Scheffers BR, Joppa LN, Pimm SL, Laurance WF (2012) What we know and don't know about Earth's missing biodiversity. Trends in Ecology \& Evolution 27 (9): 501-510. https:// doi.org/10.1016/i.tree.2012.05.008

- Shorthouse DP (2010) SimpleMappr, an online tool to produce publication-quality point maps. http://www.simplemappr.net. Accessed on: 2018-8-14.

- $\quad$ Silvertown J (2009) A new dawn for citizen science. Trends in Ecology \& Evolution 24 (9): 467-471. https://doi.org/10.1016/j.tree.2009.03.017

- $\quad$ Skvarla MJ, Tripodi A, Szalanski A, Dowling A (2015) New records of Orussus minutus Middlekauff, 1983 (Hymenoptera: Orussidae) represent a significant western range expansion. Biodiversity Data Journal 3: e5793. https://doi.org/10.3897/bdj.3.e5793

- $\quad$ Smith D (1995) Seasonal flight activity of Vanhornia eucnemidarum Crawford (Hymenoptera: Vanhorniidae) in the mid-Atlantic states. Entomological Society of Washington 97: 889-890.

- $\quad$ Suarez A, Tsutsui N (2004) The value of museum collections for research and society. BioScience 54 (1): 66-74. https://doi.org/10.1641/0006-3568(2004)054 [0066:TVOMCF]2.0.CO;2

- Thiers B, Monfils AK, Zaspel J, Ellwood E, Bentley A, Levan K, Bates J, Jennings D, Contreras D, Lagomarsino L, Mabee P, Ford L, Guralnick R, Gropp R, Revelez M, Cobb N, Lendemer J, Seltmann K, Aime MC (2019) Extending U.S. biodiversity collections to promote research and education. Biodiversity Collections Network 1-28. URL: http:// bit.ly/2XZkWKq

- Townes H, Townes M (1981) A revision of the Serphidae (Hymenoptera). Memoirs of the American Entomological Institute 32: 1-541. 


\section{Supplementary material}

Suppl. material 1: Vanhornia eucnemidarum NA Records doi

Authors: Joshua Hogan

Data type: Occurrences

Brief description: This datasheet provides an accessible way to search collection records gathered during this study.

Download file (84.84 kb) 